\title{
A New Approach For In Situ Analysis In Fully Coupled Earth System Models
}

\author{
Ufuk Utku Turuncoglu* \\ turuncu@ucar.edu \\ Climate and Global Dynamics \\ Laboratory, National Center for \\ Atmospheric Research \\ Boulder, Colorado
}

\author{
Barıs Önol \\ Aeronautics and Astronautics Faculty, \\ Meteorological Engineering, Istanbul \\ Technical University \\ Istanbul, Turkey \\ onolba@itu.edu.tr
}

\author{
Mehmet Ilicak \\ Eurasia Institute of Earth Sciences, \\ Istanbul Technical University \\ Istanbul, Turkey \\ milicak@itu.edu.tr
}

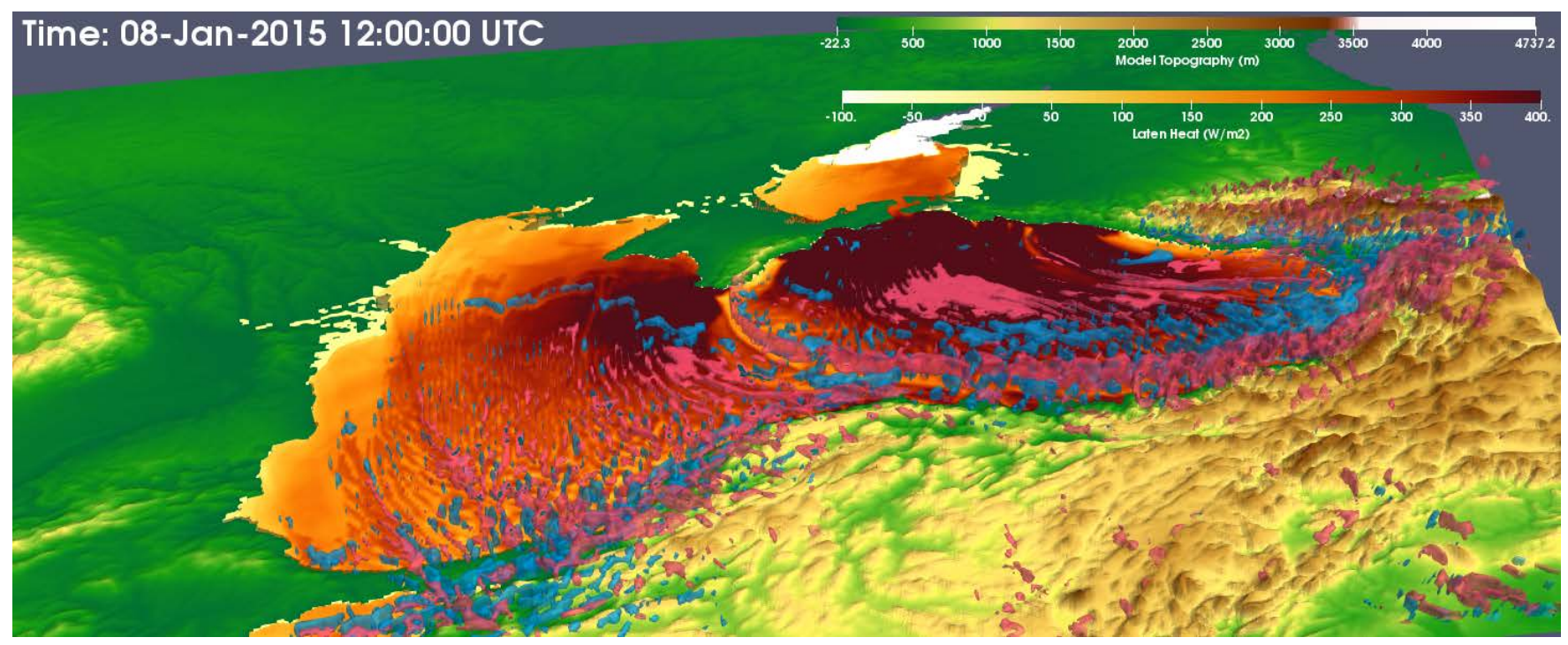

Figure 1: Iso-surface of cloud water (blue, $1 \times 10^{-6} \mathrm{~kg} / \mathrm{kg}$ ) and ice content (red, $7 \times 10^{-5} \mathrm{~kg} / \mathrm{kg}$ ) from atmosphere model with $4 \mathrm{~km}$ horizontal resolution and Latent Heat Flux $\left(\mathrm{W} / \mathrm{m}^{2}\right)$ from ocean component at $8 \mathrm{Jan}$ 2015, 12:00 UTC.

\begin{abstract}
This work focuses on enabling the use of existing in situ analysis and visualization methods within the model coupling framework, which is specially designed software layer to combine physical components of Earth system (i.e., atmosphere, ocean) through a standardized calling interface to simplify common tasks such as interpolation among different numerical grids and synchronization of the model components. The new approach enables simultaneous analysis of the vast amount of data produced by a multi-component Earth system model in an integrated way and facilitates efficient, interoperable, and optimized data analysis and visualization workflows for Earth system science applications. To demonstrate the flexibility and the capability of the new approach, a fully coupled

Permission to make digital or hard copies of all or part of this work for personal or classroom use is granted without fee provided that copies are not made or distributed for profit or commercial advantage and that copies bear this notice and the full citation on the first page. Copyrights for components of this work owned by others than the author(s) must be honored. Abstracting with credit is permitted. To copy otherwise, or republish, to post on servers or to redistribute to lists, requires prior specific permission and/or a fee. Request permissions from permissions@acm.org.

ISAV'19, November 18, 2019, Denver, CO, USA

(c) 2019 Copyright held by the owner/author(s). Publication rights licensed to ACM. ACM ISBN 978-1-4503-7723-2/19/11 ..\$15.00

https://doi.org/10.1145/3364228.3364231
\end{abstract}

regional atmosphere-ocean modeling application that aims to investigate air-sea interaction in a very high spatial and temporal resolution has been implemented. In the use case application, both the simulation and in situ visualization application run in parallel on a large-scale HPC platform with both software and hardware rendering support through the use of accelerators.

\section{CCS Concepts}

- Human-centered computing $\rightarrow$ Scientific visualization; - Computing methodologies $\rightarrow$ Massively parallel and highperformance simulations; Scientific visualization; • Applied computing $\rightarrow$ Environmental sciences.

\section{Keywords}

in situ visualization, high performance computing, earth system science, fully coupled model

\section{ACM Reference Format:}

Ufuk Utku Turuncoglu, Barıs Ȯnol, and Mehmet Ilicak. 2019. A New Approach For In Situ Analysis In Fully Coupled Earth System Models. In ISAV: In Situ Infrastructures for Enabling Extreme-Scale Analysis and Visualization (ISAV'19), November 18, 2019, Denver, CO, USA. ACM, New York, NY, USA, 6 pages. https://doi.org/10.1145/3364228.3364231 


\section{Introduction}

The complex and non-linear interaction among the physical, chemical and biological components of the Earth requires a system approach to understand the functioning and behavior of Earthrelated processes on a global and regional scale. In essence, the behavior is reflected in the definition of a "system" that is the set of interacting or interdependent components enclosed within a defined boundary. The importance of understanding Earth as an integrated system of components and processes that evolve with different spatial and temporal scales is also essential to science and education. Despite significant technological advances in highperformance computing and earth observation systems, the current understanding of the Earth components (i.e., atmosphere, ocean, land, ice) fails to fully recognize complex and multiple-scale interactions characterizing global effects of local features such as complex topography, land-sea gradients and the influence of human activities on a smaller spatial scale. Computationally demanding state-of-art modeling systems in use today either represent a limited spectrum appropriate for the particular geometry and grid or parameterize corresponding processes. It is clear that the next-generation fully coupled Earth system models (ESMs) will require new computational methods, data analysis approaches, and advanced modeling techniques to account for complex processes and data assimilated from new observation systems.

The development of the more complex and high-resolution ESMs to represent physical model components and their interaction in a more realistic way leads to an increased demand for both computational and data storage resources. In general, the high volume of data produced by the fully coupled modeling systems may not allow storing all the critical and valuable information to use later, despite recent advances in storage systems. As a result, the simulation results are stored in a limited temporal (i.e., daily, monthly averages) resolution, which is processed after numerical simulations are finished (post-processing). The poor representation of the results of numerical model simulations prevents analyzing the fast-moving processes such as extreme precipitation events, convection, turbulence, and non-linear interactions among the model components on a high temporal and spatial scale with the conventional data processing approach. Additionally, the transferring large volumes of data across relatively slow network bandwidth introduce extra bottlenecks and fail to match the ultimate demands of data processing and to archive tasks of the present high-resolution multi-component ESMs. Besides conventional data analysis approaches, the in situ visualization allows researchers to simultaneously analyze and visualize the simulation data while the numerical simulations are being run. The coupling of computation and analysis steps helps to implement efficient data analysis and visualization pipelines and boost the data analysis workflow and our understanding of underlying physical processes.

The presented work is aimed at revisiting the implementation strategy of in situ visualization for the fully coupled ESMs to explore the feasibility of using a model coupling framework to provide in situ visualization for easy-to-use, generic, standardized, and robust scientific applications of Earth system modeling. In this case, a coupling framework is defined as an environment for coupling individual model components through a standardized software interface and aims to reduce the complexity of regular tasks. These tasks include performing spatial interpolation across different computational grids, transferring data among model components, and synchronization of the execution of the model components and their interaction to increase the efficiency and interoperability of multi-component modeling systems. In this design, the in situ visualization application only interacts with the model coupling framework to retrieve, process, and analyze the data flowing through the model components. The new approach simplifies the interaction between model components and the in situ visualization application and provides new capability of interoperability and standardization to the existing ESMs and their applications. Additionally, the data produced by the multi-component ESMs can process in an integrated way to reveal a better understanding of the underlying physical processes and their non-linear interactions.

\section{Previous Work}

The existing visualization libraries such as ParaView Catalyst [9] and VisIt Libsim [22] support in situ visualization by converting the internal data structures of the simulation code into their VTK [14] equivalents. The main aim of these widely used tools is to simplify the data exchange between simulation codes and data analysis environment by providing a light-weight application programming interface (API) along with support for Python to implement flexible analysis and visualization pipelines. On the other hand, tools such as SENSEI [5] and Damaris/Viz [8] aim to provide additional software layers to take advantage of extension through the plugins to execute analysis in one of several different in situ software implementations such as ParaView Catalyst and VisIt Libsim.

Recently, several in situ visualization systems for analyzing numerical simulations of Earth system processes have been implemented. For instance, the ocean component of Model for Prediction Across Scales (MPAS) has been integrated with an image-based in situ visualization tool to examine the critical elements of the simulations and reduce the data needed to preserve those elements by creating a flexible work environment for data analysis and visualization $[1,13]$. Additionally, the same modeling system (MPAS-Ocean) has been used to study eddies in large-scale, high-resolution simulations. In this case, the in situ visualization workflow is designed to perform eddy analysis at higher spatial and temporal resolutions than available with traditional methods facing storage size and input/output bandwidth constraints [23]. Moreover, a regional weather forecast model (Weather Research and Forecasting model; WRF) has been integrated with an in situ visualization tool to track cyclones based on an adaptive algorithm [10]. Despite the lack of generic and standardized implementation for integrating model components with in situ visualization tools, the previous studies have shown that in situ visualization can produce analyses of simulation results, revealing many details in an efficient and optimized way.

On the other hand, there is no such in situ visualization application of ESMs to facilitate integrated analysis of data flowing from multiple model components at the same time. The main reason is that the multi-scale nature of the Earth system with inherently coupled and independently developed individual physical model 
components that make the integration of in situ visualization application with the modeling system challenging. In this case, each model component needs to interact with the underlying in situ visualization application separately due to the difference of the used data structures, underlying numerical grid representation (i.e., unstructured, curvilinear), and parallel two/three-dimensional domain decomposition configuration in the model components. It is evident that more generic in situ visualization implementation for Earth system science could facilitate smooth integration of the existing standalone and coupled ESMs with available in situ visualization tools $[2,4,6,7]$ and improve interoperability between such tools and non-standardized numerical simulation codes.

\section{Design and Implementation}

The newly designed in situ visualization aware coupled modeling system uses ParaView/Catalyst API to develop a "unified adaptor" code for ESM applications (Fig. 2). The reason for using "unified adaptor" as a term to define the wrapper layer between model and co-processing plugin is that the developed adaptor code is independent of implementation details of the physical model component (i.e., data structures, numerical grid, and domain decomposition type) and uses standardized software interface to interact with the individual models. In this design, the adaptor acts as a wrapper layer and transforms information coming from the model component to the co-processing plugin in a compatible form and facilities to process data coming from different model components.

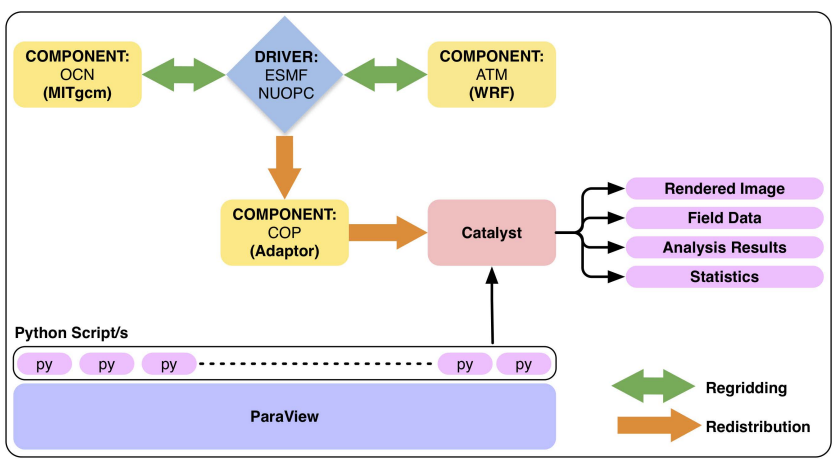

Figure 2: Design of the modeling system: model components and their interactions (orange arrows represent the redistribution and green arrows represent interpolation).

With this approach, the modeling systems, which are coupled with the Earth System Modeling Framework (ESMF) library [17] and the National Unified Operational Prediction Capability (NUOPC) layer, may benefit from the use of an integrated modeling framework to analyze the data flowing from the multi-component modeling system without extensive code development and restructuring. The NUOPC layer simplifies component synchronization and run sequence by providing an additional programming interface between the coupled model and ESMF through the use of a NUOPC "cap". In this case, a NUOPC "cap" is a Fortran module that serves as the interface to a model when it is used in a NUOPC-based coupled system. The term "cap" is used because it is a small software layer that sits on top of a component code, making calls into it and exposing model data structures in a standard way.
The design of the three-component coupled system that is used in this study can be seen in Fig.2. In this case, the synchronization of the model components is handled by the driver component, which is developed by ESMF/NUOPC API interface. The driver is the component that is responsible for the orchestration of the overall modeling system resides in the middle and acts as a translator among model components, and there is no direct interaction among model components, including co-processing. In this design, the adaptor code interacts with the driver through the use of the NUOPC cap and provides an abstraction layer for the ParaView/Catalyst. The adaptor code is designed to apply multiple visualizations and dataprocessing pipelines (Python scripts) to the simulation results at each coupling time step to make a different set of analyses over the results of the numerical simulation for more efficient data analysis without rerunning simulation (Fig.2). The modeling system also facilitates multiple input ports to process data flowing from multiple physical model components. The input ports are defined automatically by the co-processing component based on activated model components and each model component has two ports to handle both two and three-dimensional fields separately such as atm_input2d, atm_input3d, ocn_input $2 d$ and ocn_input $3 d$.

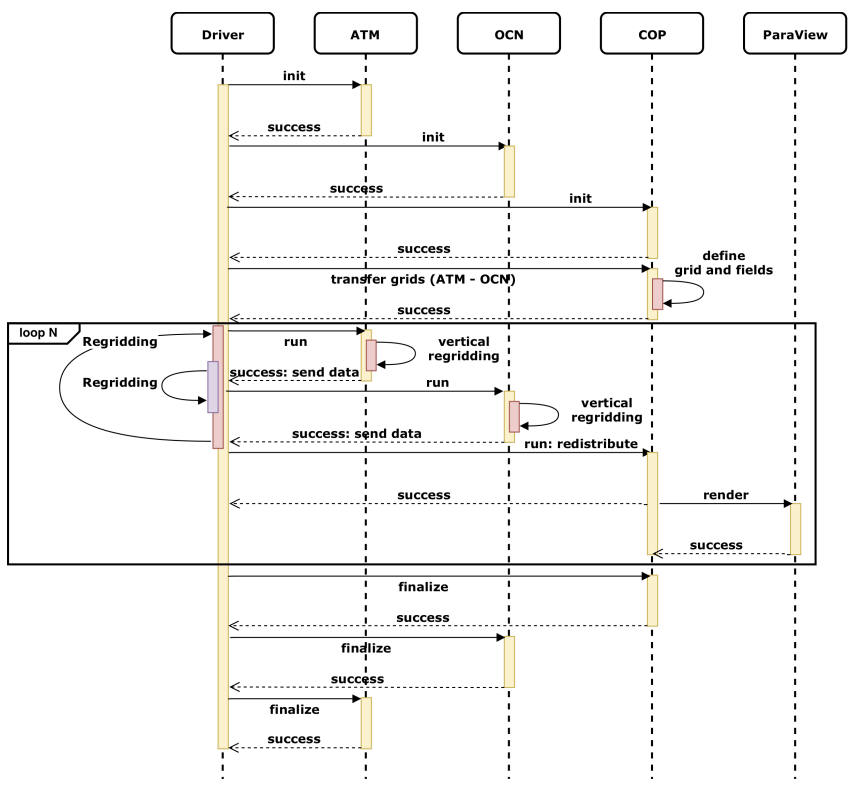

Figure 3: Sequence diagram for newly designed RegESM modeling system.

In the initialization phase of the modeling systems, the numerical grid of physical model components (atmosphere; ATM and ocean; $\mathrm{OCN}$ ) is transferred to the co-processing component (COP) to transform underlying grid representations into their VTK equivalents using adaptor code (Fig. 3). In this case, the ESMF grid object is used to create a vtkStructuredGrid along with their modified parallel two-dimensional decomposition configuration, which is supported by the ESMF/NUOPC grid transfer capability. The initialization phase is also responsible for defining exchange fields and mapping ESMF_Field data structure as vtkMultiPieceDataSet objects in the COP component. The run phase includes a time-stepping loop to 
run the model component in a defined period and continues until the simulation ends. The time interval to exchange data between the model and co-processing component is defined using a configurable namelist option (coupling time step), just like the interaction among other model components. In each coupling loop, the driver component prepares exchange fields according to the interaction among components by applying interpolation (except coupling with the co-processing component), performing a unit conversion and common operations over the fields such as rotation of wind field due to the mismatch between the ocean and atmosphere model grids. During the simulation, the COP component also synchronizes with the simulation code and retrieves updated data to process the results at each coupling time interval, and the driver continues to redistribute the exchange fields using ESMF_FieldRedist calls (Fig. 3). The NUOPC cap of physical model components also supports vertical interpolation of the three-dimensional fields from terrain-following coordinates of the WRF model to height coordinates before passing information to the COP component. In this design, the vertical interpolation is introduced to have consistency in the vertical scales and units of the data coming from model components. Then, finalizing routine of the components is called to stop the model simulations and destroy the defined data structure(s) and free the memory (Fig. 3).

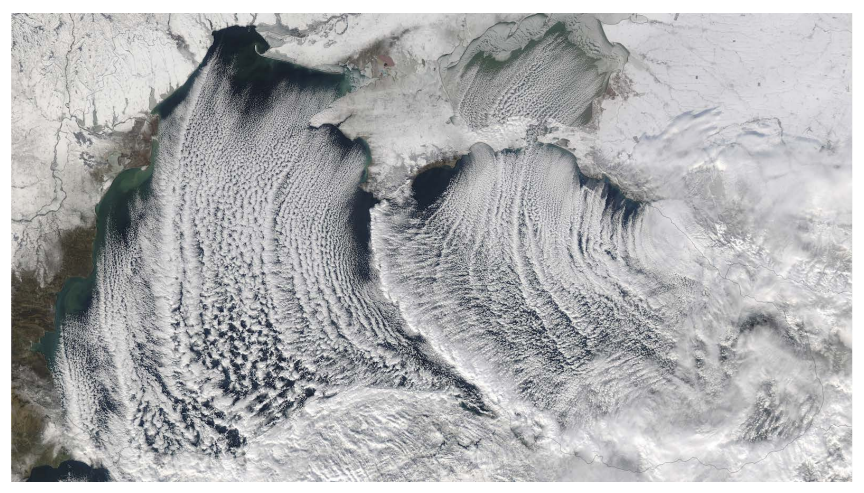

Figure 4: The Moderate Resolution Imaging Spectroradiometer (MODIS) on the Aqua satellite captured image of cloud streets over the Black Sea in January 2015.

To demonstrate the capability of the newly designed integrated modeling system described above, the three-component high-resolution configuration of RegESM (version 1.2) regional modeling system is implemented to analyze air-sea interaction and evolution of cloud streets over the Black Sea on January 8, 2015 (Fig. 4). Cloud streets are long parallel bands of cumulus clouds that form when cold air blows over warmer waters, and a warmer air layer (temperature inversion) rests over the top of both, and the comparatively warm water gives up heat and moisture to the cold air above [21]. The strength of the snow and rainfall strongly dependent on the temperature difference between air and water, wind direction, and wind speed and understanding of air and sea interaction and the underlying physical processes has vital importance to predict such extreme events. The modeling system is a completely redesigned version of the previously used and validated coupled modeling system to study the regional climate of the Caspian [19]. The version that is used in this study is also an extended version of the previously demonstrated in situ visualization enabled modeling system to investigate the evolution of Hurricane Katrina [18] and climate of Mediterranean Basin [20]. In this case, two components are modified to allow coupling with co-processing component: (1) the Advanced Research WRF model (ARW; [15]), which is developed and sourced from National Center for Atmospheric Research (NCAR), and (2) MIT general circulation model (MITgcm c63s; [11, 12]).

The WRF model is configured to include three nested atmospheric model domains. In this case, a widely used offline-nesting approach is used in the transition from low-resolution to high-resolution models to provide lateral boundary conditions for finer domains. The outer most model domain with a resolution of $12 \mathrm{~km}$ (D1) is centered in Europe and covers almost entire Europe to provide required atmospheric state variables to the inner model domains to allow better representation of the large-scale atmospheric circulation systems and uses ECMWF's ERA5 reanalysis data as lateral boundary and initial condition. The horizontal grid spacing of the second (D2) and third (D3) inner nest domains are defined as $4 \mathrm{~km}$ (625x465x61) and $1 \mathrm{~km}(1483 \times 1186 \times 61)$ respectively, and both of them cover the entire Black Sea to provide high-resolution atmospheric forcing to the ocean model for fully coupled simulations. All domains have 61 vertical terrain-following sigma layers to better resolve the simulated microphysical and the planetary boundary layer $(\mathrm{PBL})$ processes in the vertical. The internal time step is set to 20 -seconds for the D2 domain and 3-seconds for D3 domains of the atmosphere model.

The MITgcm ocean model is configured to cover the entire Black Sea to provide sea surface temperature (SST) to the atmosphere model as a surface boundary condition. The ocean model has a spatial resolution of $1 / 72^{\circ}$, which corresponds to a non-uniform resolution of around $1.5 \mathrm{~km}(896 \times 440$ grid points). The model has 92 depth layers to provide a detailed representation of the main circulation patterns of the region and vertical tracer gradients. The bottom topography data of the model is constructed using the ETOPO1 dataset [3], and the minimum depth is set to $10 \mathrm{~m}$. The initial condition of the ocean model is derived from the Black Sea Physics Reanalysis Product provided by COPERNICUS Marine Environment Monitoring Service. The internal time step of the ocean model component is defined as 30 -seconds. The interaction between model components is defined as 3-minutes to analyze the model results in a very high temporal resolution.

\section{Performance benchmark}

A set of simulations is performed with the different model configurations to assess the overall performance of the coupled modeling system by focusing on the overhead of the COP component. To isolate the overhead of the driver component itself from the overhead of the co-processing component, first individual model components (ATM and OCN) are run in standalone mode, and then two-component (ATM-OCN) and three-component cases (ATM$\mathrm{OCN}-\mathrm{COP}$ ) are run to collect timing information. In this case, the simulation length is configured as 3-hours for both standalone and coupled simulations, and the coupling interval is set to 3-minutes. In the benchmark simulations, the model components are configured not to write checkpoint and history data to eliminate I/O overhead 
of the model component from the timing results (Table 1). However, an additional test is designed to measure I/O overhead of the COP component in the case of writing data in VTK format. The model simulations and performance benchmarks are done on a Cheyenne cluster provided by the NCAR-Wyoming Supercomputing Center. Due to the lack of GPU accelerators in the entire system, the benchmark simulations are done using ParaView 5.6.1 build with Mesa library (version 17.3.9) for software-based rendering.

Table 1 shows the timing (s) and overhead (\%) results for two different domains (D2 and D3) and their various configurations. The comparison of the standalone and two-component coupled model configuration reveals that the coupling of physical model components introduces an additional \%5-6 overhead in the total execution time, which increases to $\% 21.5$ in low-resolution case (D2) for a large number of MPI tasks. It is also considered as the overhead of the driver component. The main reason for seeing relatively high overhead in the lower resolution simulation is the relatively small problem size and the increased MPI (Message Passing Interface) communications that dominate the overall performance of the modeling system. The benchmark results also reveal that the three-component case (ATM-OCN-COP) adds extra \%5 overhead for I/O and \%7 for rendering in high-resolution cases (D3) when it is compared with the two-component case. In low-resolution case, the overhead of the COP component reaches around $\% 10$ for rendering and $\% 20$ for I/O cases for low MPI task count. The results show that the time spent in the co-processing component is acceptable, especially for high-resolution cases and, does not introduce any significant performance pitfalls in the current configuration. It is also noted that the time spends on the co-processing component also depends on the designed visualization pipeline and the number of fields and dimensions.

Table 1: Rendering and I/O wall-clock time (s) and overhead (\%) for tested model configurations.

\begin{tabular}{|l|c|c|c|}
\cline { 2 - 4 } \multicolumn{1}{c|}{} & \multicolumn{2}{c|}{ Domain 2) } & Domain 3 \\
\hline \# cores & $\mathbf{2 8 0}$ & $\mathbf{1 2 8 0}$ & $\mathbf{1 2 8 0}$ \\
\hline ATM & 303 & 110 & 2628 \\
\hline OCN & 110 & 61 & 61 \\
\hline ATM-OCN & $435(\% 5.05)$ & $218(\% 21.5)$ & $2868(\% 6.2)$ \\
\hline ATM-OCN-COP (I/O) & $557(\% 25.9)$ & $426(\% 59.9)$ & $3028(\% 11.2)$ \\
\hline ATM-OCN-COP (Rendering) & $487(\% 15.2)$ & $351(\% 51.3)$ & $3094 \% 13.1)$ \\
\hline
\end{tabular}

\section{Results}

Figure 1 shows snapshots of the model-simulated cloud water and ice content iso-surfaces that are generated by processing the three-dimensional atmosphere model output from $4 \mathrm{~km}$ horizontal resolution model output. The analysis of simulations reveals that the water and ice content of clouds shows higher spatial variability in high-resolution simulation (not shown here), and the overall structure of the cloud ice content shows different spatial patterns, especially over the eastern part of the Black Sea. The low and high-resolution simulations reveal that a large fraction of rainfall comes from squall lines that are formed over the northern coast of Turkey and propagates along with the coastline. This result consistent with the observations and satellite imaginary showed in Fig. 3. The test with increased sea surface temperature provided by the ocean model component also reveals that the increased sea surface temperature leads to an increase of cloud water vapor in the lower level atmosphere, which is associated with the rainfall along the coastal part of northern Turkey. The initial results also reveal the strong relationship between sea surface temperature anomaly and atmospheric moisture content and also observed in the ocean model output, especially in latent heat flux, which is an indicator of moisture flux from the ocean to the atmosphere. Fig.1.

\section{Conclusion and Future Work}

In this study, the added value of integrating the Earth System Modeling Framework with an in situ visualization and analysis tool is demonstrated. The results show that the newly developed modeling system provides easy to use and generic numerical simulation environment for ESM applications along with the help of "unified adaptor" layer. The new design also promotes the usage of coprocessing capability with the existing earth system models, which is coupled using the ESMF framework and NUOPC layer to analyze a large volume of data in a very high temporal resolution and integrated way. The usage of in situ visualizations also helps to reduce the amount of stored data of a very high-resolution model configuration. The main drawback of the current implementation is that a priori knowledge needed about the underlying physical mechanism to reveal added value information about the interaction among the ESM components and design the visualization pipeline. Besides, the overall performance of the modeling system is affected by the co-processing component, especially for the software rendering case because of the tightly integrated simulation and co-processing components design. Additionally, the implemented co-processing component can be extended by using SENSEI or Damaris/Viz to support different in situ visualization tools along with the usage of the plugin approach. Moreover, the support for the loosely coupled modeling paradigm, using a separate set of visualization nodes for processing, can also be implemented to support a wide variety of HPC platforms. However, it will require a restructuring of the newly designed modeling system to support multiple executables in which the ESMF library has limited support through the use of InterComm [16].

\section{Acknowledgments}

Computing and data storage resources, including the Cheyenne (DOI:10.5065/D6RX99HX) and Casper supercomputers, were provided by the Computational and Information Systems Laboratory (CISL) at NCAR under grant SCSG0002 (PI, Siddhartha Ghosh). This material is based upon work supported by the NCAR, which is a facility sponsored by the National Science Foundation under Cooperative Agreement No. 1852977. This study has also been supported by a research grant (116Y136) provided by The Scientific and Technological Research Council of Turkey (TUBITAK). The NVIDIA Corporation donated the Quadro K5200 as part of the Hardware Donation Program. The author extends his thanks to John Clyne and Scott Pearse from NCAR/CISL, Rocky Dunlap and Robert Oehmke from NCAR/CSEG, Gerhard Theurich from Science Applications International Corporation (McLean, Virginia), Andy C. Bauer from Kitware Inc., USA, and Mahendra Roopa from NVIDIA for their very useful suggestions and helps. 


\section{References}

[1] James Aherns, Sebastien Jourdain, Patrick O'Leary, John Patchett, David Rogers, Patricia Fasel, Andrew Bauer, Mark Petersen, Francesca Samsel, and Benjamin Boeckel. 2014. In Situ MPAS-Ocean Image-Based Visualization. In Proceedings of the International Conference for High Performance Computing, Networking, Storage and Analysis.

[2] James Paul Ahrens and Berk Geveci. 2005. ParaView: An End-User Tool for Large-Data Visualization. In The Visualization Handbook.

[3] Christopher Amante and B. Eakins. 2009. ETOPO1 1 Arc-Minute Global Relief Model: procedures, data sources and analysis. (03 2009). https://doi.org/10.7289/ V5C8276M

[4] Utkarsh Ayachit. 2015. The ParaView Guide: A Parallel Visualization Application. Kitware, Inc., USA.

[5] Utkarsh Ayachit, Brad Whitlock, Matthew Wolf, Burlen Loring, Berk Geveci, David Lonie, and E. Wes Bethel. 2016. The SENSEI Generic in Situ Interface. In Proceedings of the 2Nd Workshop on In Situ Infrastructures for Enabling Extremescale Analysis and Visualization (ISAV '16). IEEE Press, Piscataway, NJ, USA, 40-44. https://doi.org/10.1109/ISAV.2016.13

[6] E. Wes Bethel, Hank Childs, and Charles Hansen. 2012. High Performance Visualization: Enabling Extreme-Scale Scientific Insight (1st ed.). Chapman \& Hall/CRC.

[7] Hank Childs, Eric Brugger, Brad Whitlock, Jeremy Meredith, Sean Ahern, Kath leen Bonnell, Mark Miller, Gunther H. Weber, Cyrus Harrison, Thomas Fogal, Christoph Garth, Allen S, E. Wes Bethel, Marc Durant, David Camp, Jean M. Favre, Oliver Rubel, Paul Navratil, Matthew Wheeler A, Paul Selby, and Fabien Vivodtzev. 2011. VisIt: An End-User Tool For Visualizing and Analyzing Very Large Data. In In Proceedings of SciDAC

[8] M. Dorier, R. Sisneros, T. Peterka, G. Antoniu, and D. Semeraro. 2013. Damaris/Viz: A nonintrusive, adaptable and user-friendly in situ visualization framework. In 2013 IEEE Symposium on Large-Scale Data Analysis and Visualization (LDAV) 67-75. https://doi.org/10.1109/LDAV.2013.6675160

[9] Nathan Fabian, Kenneth Moreland, David Thompson, Andrew C. Bauer, Pat Marion, Berk Gevecik, Michel Rasquin, and Kenneth E. Jansen. 2011. The ParaView Coprocessing Library: A scalable, general purpose in situ visualization library. In 2011 IEEE Symposium on Large Data Analysis and Visualization. 89-96. https://doi.org/10.1109/LDAV.2011.6092322

[10] Preeti Malakar, Vijay Natarajan, and Sathish S. Vadhiyar. 2012. Integrated Parallelization of Computations and Visualization for Large-scale Applications. In Proceedings of the 2012 IEEE 26th International Parallel and Distributed Processing Symposium Workshops \& PhD Forum (IPDPSW '12). IEEE Computer Society, Washington, DC, USA, 2510-2513. https://doi.org/10.1109/IPDPSW.2012.314

[11] John Marshall, Alistair Adcroft, Chris Hill, Lev Perelman, and Curt Heisey. 1997. A finite-volume, incompressible Navier Stokes model for studies of the ocean on parallel computers. Journal of Geophysical Research: Oceans 102, C3 (1997), 5753-5766. https://doi.org/10.1029/96JC02775

[12] John Marshall, Chris Hill, Lev Perelman, and Alistair Adcroft. 1997. Hydrostatic quasi-hydrostatic, and nonhydrostatic ocean modeling. Fournal of Geophysical Research: Oceans 102, C3 (1997), 5733-5752. https://doi.org/10.1029/96JC02776

[13] Patrick O’Leary, James Ahrens, Sébastien Jourdain, Scott Wittenburg, David H. Rogers, and Mark Petersen. 2016. Cinema image-based in situ analysis and visualization of MPAS-ocean simulations. Parallel Comput. 55 (2016), $43-48$ https://doi.org/10.1016/j.parco.2015.10.005 Visualization and Data Analytics for Scientific Discovery.

[14] William J. Schroeder, Kenneth M. Martin, and William E. Lorensen. 1996. The Design and Implementation of an Object-oriented Toolkit for 3D Graphics and Visualization. In Proceedings of the 7th Conference on Visualization '96 (VIS '96). IEEE Computer Society Press, Los Alamitos, CA, USA, 93-ff. http://dl.acm.org/ citation.cfm?id=244979.245018

[15] William C. Skamarock, Joseph B. Klemp, Jimy Dudhia, David O. Gill, Zhiquan Liu, Judith Berner, Wei Wang, G. Jordan Powers, Michael G. Duda, Dale M. Barker, and Xiang-Yu Huang. 2019. A Description of the Advanced Research WRF Model Version 4. Technical Report. https://doi.org/10.5065/1dfh-6p97

[16] Alan Sussman. 2006. Building complex coupled physical simulations on the grid with InterComm. Engineering with Computers 22, 3 (01 Dec 2006), 311-323. https://doi.org/10.1007/s00366-006-0037-7

[17] Gerhard Theurich, C. DeLuca, T. Campbell, F. Liu, K. Saint, M. Vertenstein, J Chen, R. Oehmke, J. Doyle, T. Whitcomb, A. Wallcraft, M. Iredell, T. Black, A. M. Da Silva, T. Clune, R. Ferraro, P. Li, M. Kelley, I. Aleinov, V. Balaji, N. Zadeh, R. Jacob, B. Kirtman, F. Giraldo, D. McCarren, S. Sandgathe, S. Peckham, and R. Dunlap. 2016. The Earth System Prediction Suite: Toward a Coordinated U.S Modeling Capability. Bulletin of the American Meteorological Society 97, 7 (2016), 1229-1247. https://doi.org/10.1175/BAMS-D-14-00164.1

[18] Ufuk U. Turuncoglu. 2019. Toward modular in situ visualization in Earth system models: the regional modeling system RegESM 1.1. Geoscientific Mode Development 12, 1 (2019), 233-259. https://doi.org/10.5194/gmd-12-233-2019

[19] U. U. Turuncoglu, G. Giuliani, N. Elguindi, and F. Giorgi. 2013. Modelling the Caspian Sea and its catchment area using a coupled regional atmosphere-ocean model (RegCM4-ROMS): model design and preliminary results. Geoscientific
Model Development 6, 2 (2013), 283-299. https://doi.org/10.5194/gmd-6-283-2013

[20] Ufuk Utku Turuncoglu and Gianmaria Sannino. 2017. Validation of newly designed regional earth system model (RegESM) for Mediterranean Basin. Climate Dynamics 48, 9 (01 May 2017), 2919-2947. https://doi.org/10.1007/ s00382-016-3241-1

[21] Adam Voiland. 2019. Cloud Streets over the Black Sea. Retrieved July 21, 2019 from https://earthobservatory.nasa.gov/images/85056/ cloud-streets-over-the-black-sea

[22] Brad Whitlock, Jean M. Favre, and Jeremy S. Meredith. 2011. Parallel In Situ Coupling of Simulation with a Fully Featured Visualization System. In Eurographics Symposium on Parallel Graphics and Visualization, Torsten Kuhlen, Renato Pajarola, and Kun Zhou (Eds.). The Eurographics Association. https: //doi.org/10.2312/EGPGV/EGPGV11/101-109

[23] Jonathan Woodring, Mark Petersen, Andre Schmeiber, John Patchett, James Ahrens, and Hans Hagen. 2016. In Situ Eddy Analysis in a High-Resolution Ocean Climate Model. IEEE Transactions on Visualization \& Computer Graphics 22, 01 (jan 2016), 857-866. https://doi.org/10.1109/TVCG.2015.2467411 